East African Medical Journal Vol. 86 No. 6 June 2009

VERTEX EPIDURAL HAEMATOMA MANIFESTING WITH BILATERAL UPPER LIMB DECEREBRATE POSTURE: CASE REPORT

J. G. Kiboi, MBChB, MMed (Surg), Neurosurgery Fellowship(Pretoria, PAH, SA), Lecturer, Department of Surgery, and

I. M. Muriithi, MBChB, MMed (Diagnostic Radiology), Tutorial Fellow, Department of Diagnostic Imaging and Radiation

Medicine, College of Health Sciences, University of Nairobi, P. O. Box 19676-00202, Nairobi, Kenya

Request for reprints to: Dr. I. M. Muriithi, P.O. Box 2479-00202, Nairobi, Kenya

\title{
VERTEX EPIDURAL HAEMATOMA MANIFESTING WITH BILATERAL UPPER LIMB DECEREBRATE POSTURE: CASE REPORT
}

\author{
J. G. KIBOI and I. M. MURIITHI
}

\begin{abstract}
SUMMARY
Vertex epidural haematomas (VEDH) are rare and difficulties are encountered in diagnosis and management. This is a case report of a patient with a vertex epidural haematoma who presented with signs of severe head injury with upper limb decerebrate posture. We discuss the challenges of radiological investigation and neurosurgical management of VEDH.
\end{abstract}

\section{INTRODUCTION}

Vertex epidural haematomas are a rare but wellknown entity. Their clinical presentation may be misleading and non-specific and may be missed by routine CT scanning resulting in delayed diagnosis with likely fatal consequences. We present a patient with a traumatic VEDH who presented unconscious with upper limb decerebrate posture, the haematoma evident at $\mathrm{CT}$ and successfully drained at surgery. We stress the importance of multiplanar CT reformatted images and a novel approach to the surgery performed.

\section{CASE REPORT}

A 46 year old male patient was admitted on $14^{\text {th }}$ September 2007 to a local hospital as a referral from a nursing home. The patient had been found unconscious in his house after missing from his work place for three days. The last time he had been seen was in a local bar on a drinking spree after which no one knew how he reached his house. The relatives broke into his house three days after he went missing and found him sprawled on the floor unconscious. There was no reported history of vomiting or convulsions. On admission, the patient was unconscious with a Glasgow coma scale of $7 / 15$ (motor 4 , eye 2, verbal 1 ). His blood pressure was $160 / 100 \mathrm{mmHg}$ and pulse rate of 70 beats per minute. His temperature was normal but he was sweating profusely. His upper limbs were extended at the elbow and the shoulder joint. The knees and hip joints were in flexion bilaterally. The pupils were bilaterally equal at $3 \mathrm{~mm}$ and sluggishly reacting to light. The muscle bulk was normal in the upper and lower limbs. The tone was increased in both the upper and lower limbs. The knee jerk and elbow reflexes were increased. A CT scan of the brain done revealed a heterogeneously hyperdense, non-enhancing, bilateral frontoparietal, extra-axial collection at the vertex displacing the superior sagittal sinus inferiorly and compressing the adjacent parasagittal portions of the cerebral hemispheres (Figures 2A and 3). A right parietal haemorrhagic contusion was also noted. Cerebral veins were shown draining into the superior sagittal sinus bilaterally (Figures 2A and 3B). Images in bone window settings revealed a bilateral linear parietal fracture at the vertex (Figure 2B). Reconstruction coronal and sagittal CT images clearly demonstrated the large vertex epidural haematoma and the superior sagittal sinus (SSS) displaced downwards away from the inner table of the calvarium (Figure 3). The craniotomy was done after thorough planning that involved designing the simplest approach with the aim of hitching the dura while safely lifting up the superior sagittal sinus. Taking into account the blood supply to the scalp from the superficial temporal artery, bilateral inverted scalp U-flaps were raised with a midline sagittal incision and the scalp flaps retracted laterally (Figure 1). 


\section{Figure $1 \mathrm{~A}$ and $\mathrm{B}$}
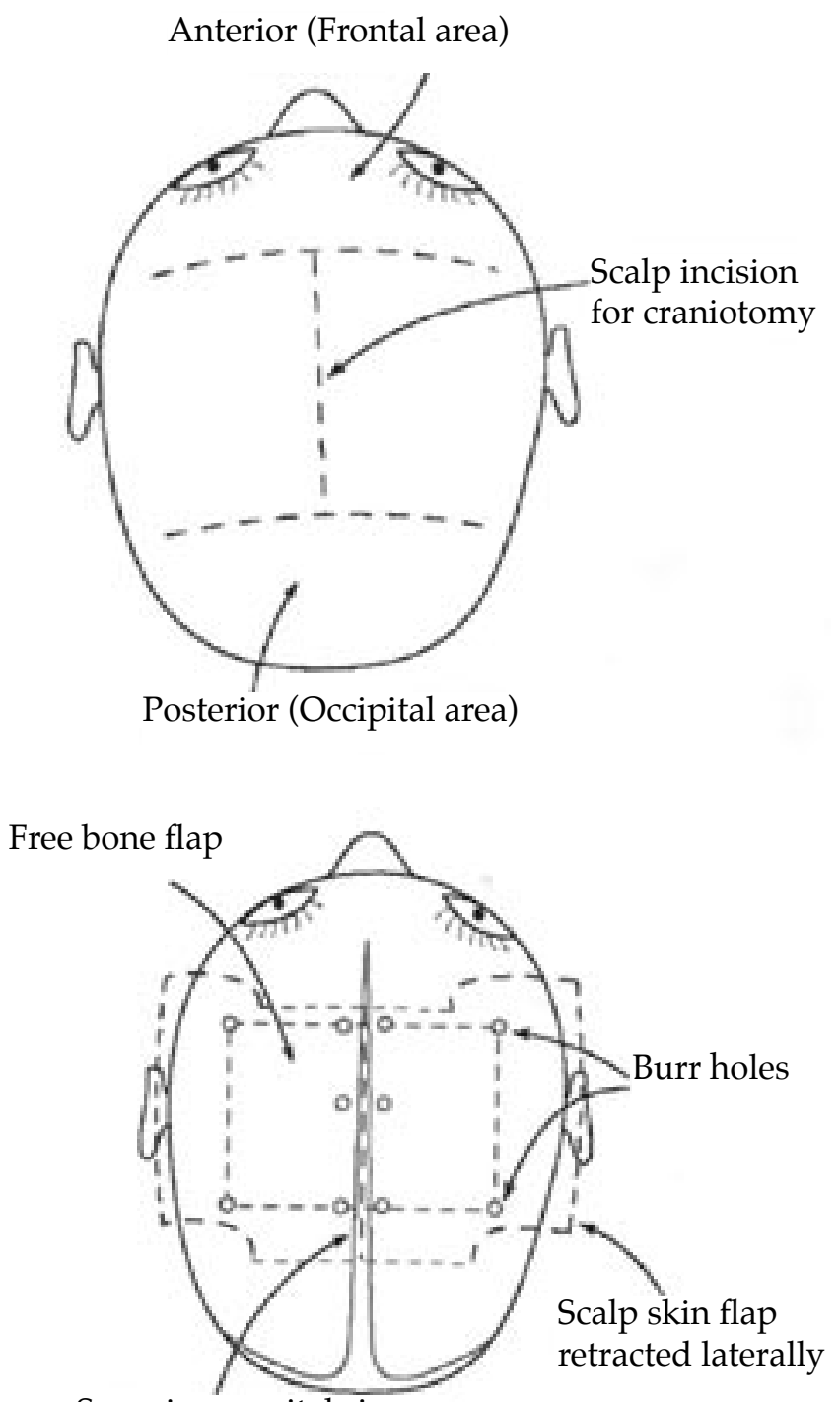

Superior saggital sinus

Figure 2

A. Post contrast axial CT Scan showing a high attenuation area at the vertex crossing the midline. Cerebral veins (arrows) are also shown draining into the midline superior sagittal sinus. B. Axial CT image in bone window setting showing the bilateral linear parietal fracture (open arrow) at the vertex
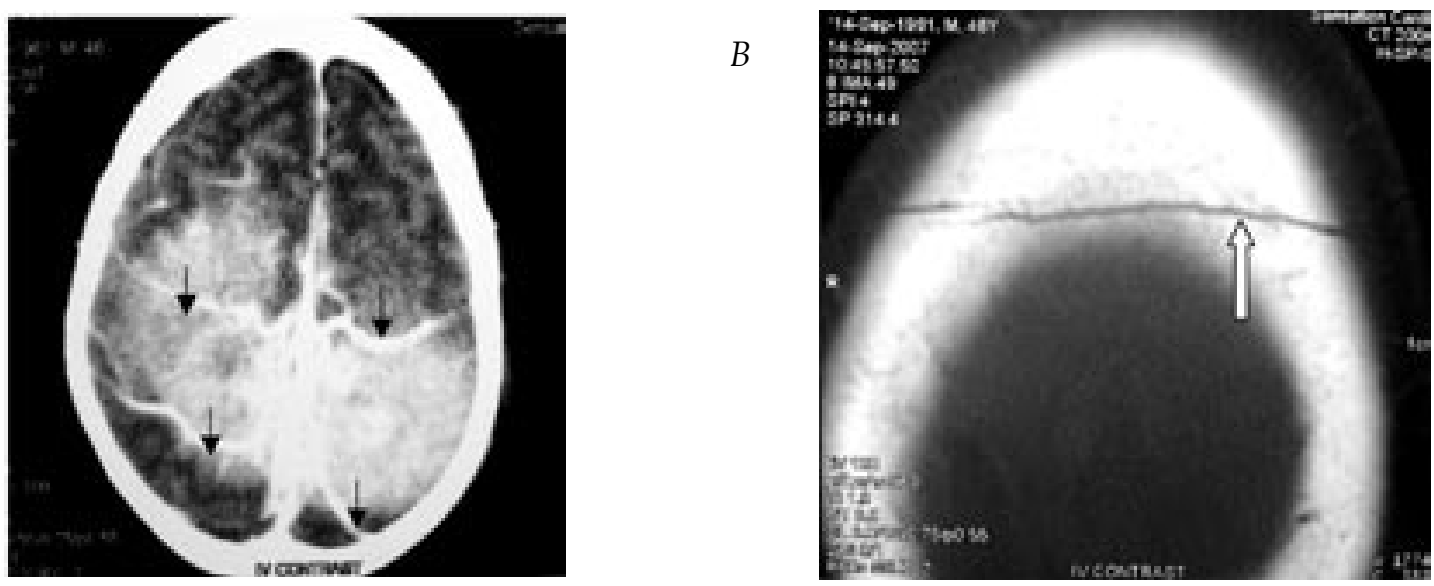


\section{Figure 3}

Left parasagittal $(A)$ and coronal $(B)$ post contrast $C T$ refomatted images better demonstrating the VEDH (straight open arrow), the extent of the superior sagittal sinus (curved open arrow) displacement and the brain compression.

A

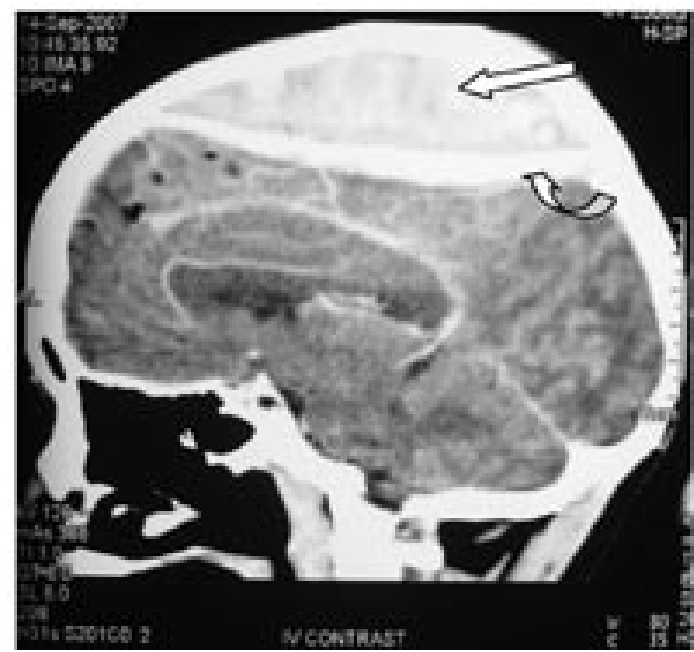

B

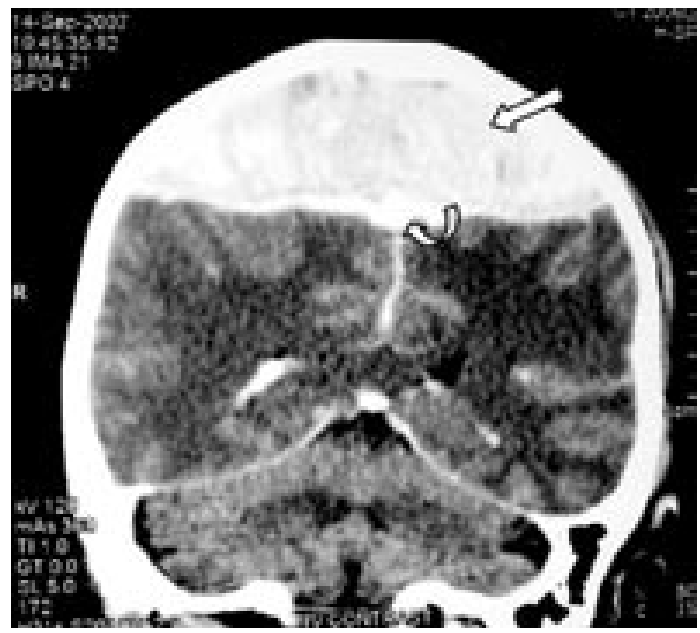

Since the superior sagittal sinus had been displaced caudally, burr hole drilling revealed the vertex haematoma easily without worry of superior sagittal sinus injury. Multiple paramidline burr holes were also made to facilitate hitching of the dura around the superior sagittal sinus. A biparietal free bone flap was then raised from the vertex uneventfully, the dura hitched initially around the craniotomy edges prior to evacuation of the haematoma. The haematoma was then evacuated but it was crucial to leave haematoma around the superior sagittal sinus to prevent complicated venous bleeding while the rest of the clot was evacuated. A 20mm needle, vicryl 3.0 suture was then used to hitch the dura around the superior sagittal sinus and sutures passed through the paramidline burr holes in the free bone flap.
Once haemostasis was confirmed and the bone flap replaced, the free paramidline sutures were then tied lifting up the midline superior sagittal sinus dural region. The scalp wound was then sutured in two layers (galea and skin) and a Potovac drain left in the pericranium surface. The wound was then dressed and the patient transferred to ICU where he was intubated and sedated for 48 hours. Postoperative haemoglobin was $6.8 \mathrm{~g} / \mathrm{dl}$ with platelets of 100 $\mathrm{x} 10^{9} / 1$. He was then transfused two pints of blood. After 48 hours the patient was responding well to commands, had good respiratory effort with good oxygen saturation levels hence he was extubated and observed overnight in ICU. On $18^{\text {th }}$ September 2007 he was opening his eyes spontaneously and had verbal response but remained confused. He was moving all the four limbs spontaneously and purposefully. He was managed in the ward from $18^{\text {th }}$ September 2007 and mobilised until he was discharged on $23^{\text {rd }}$ September 2007 after very good recovery.

\section{DISCUSSION}

Vertex epidural haematoma is a rare clinical entity that presents with challenging management problems. The causative factor in this patient was not clear clinically since there were no bruises or injuries noted on the head. Most of the reported cases of VEDHs are caused by traumatic injury (1).

The clinical course may be acute or chronic (1). Between 1964 and 1976, Borozone et al (7) treated 14 cases of vertex epidural haematomas. Eight cases had acute course with a symptom free clinical interval. Stevenson et al (8) reported a patient who was free of symptoms for six weeks but stated that most vertex epidural haematomas have acute course. However symptom free intervals have varied from hours to weeks to as long as 11 months in one case (1). In our reported case, the patient had been ill for three days and this probably was an acute course. The clinical course is not always acute and the non-specific and non-localising character may lead to a delay in therapeutic measures which may even result in unexpected death $(2,9)$.

A severe headache is the leading symptom reported (7). However, intractable vomiting, sensory complaints, nuchal rigidity, central paraplegia, papilloedema, anisocoria, visual changes and seizures have also been reported (1). Many authors report headache, vomiting, and increased intracranial pressure as the major clinical symptoms. Paraplegia and motor weakness are also important manifestations $(1,2,4)$. Our patient had no reported history of vomiting prior to admission despite the obvious increased intracranial pressure.

In a case report by Messori et al (6) a patient reported in an emergency department with paraplegia 
but was alert, had no headache or vomiting though reported some transient diminution of consciousness. Initial management was focused on spinal injury. An MRI of the spine was performed that showed no abnormality. However due to the absence of sensory disturbances and presence of striking leg hypotonia the correct diagnosis might have been suspected initially. The reported transient diminution of consciousness likewise supported the correct clinical diagnosis. Our reported patient was unconscious with a GCS of 7 and had quadriparesis with extension of the upper limbs (decerebrate posture) and fixed flexion of the knees.

In the Messori et al. (6) case the paraplegia could be explained due to pressure on the motor cortex at the vertex subserving the hips, knees and feet by the haematoma. In our patient the upper limbs decerebrate posture was probably due to increased intracranial pressure by the haematoma causing prolonged cerebral and upper brainstem compression. Decerebrate posture results from damage to the upper brainstem. In this posture, the upper limbs are adducted and extended with the wrists pronated and the fingers flexed. The lower limbs are stiffly extended with plantarflexion of the feet. However, in this patient only the upper limbs presented in decerebrate position but the lower limbs were flexed at the hips and knees. Decerebrate posture is usually a late sign of increased intracranial pressure in head trauma patients. This correlated well with the three day delay in presentation post trauma in our patient.

Majority of VEDHs are associated with cranial trauma, with various studies showing as many as 92-100\% having a calvarial fracture at the vertex (1-5). In this reported case, the CT scan revealed a bilateral parietal calvarial fracture (Figure 2B). Due to the rare nature of this lesion, routine imaging techniques may fail to show it, even in situations where a definite diagnosis of cranial trauma is present. CT scanning, which has become the examination of choice in patients with head trauma, may miss some VEDHs. Routine CT scanning in the conventional axial plane may cause bone volume-averaging effects at the vertex (due to relative thickness of CT sections $(10 \mathrm{~mm})$ on routine scanning) resulting in relatively small VEDHs being misinterpreted as artifacts or being overlooked (2,5-7).

Several authors have emphasized magnetic resonance imaging (MRI) as a much more sensitive tool for VEDH detection due to its multiplanar capability and lack of bone artifacts (5). MR combined with cerebral magnetic resonance venography (MRV) can also be useful to determine severity of SSS displacement and assess for SSS thrombosis or laceration resulting from diastatic fractures $(5,8)$.
However, Saffavi-Abbasi et al (1) suggest that coronal CT scanning or coronal reconstructions are preferable and sufficient in all clinically suspected cases of VEDHs. Coronal and sagittal reconstructions allow detection of even small VEDHs (6). Coronal and sagittal CT reconstructed images better demonstrated the actual extent of the vertex haematoma in our patient and further characterised the displaced superior sagittal sinus (Figure 3). Coronal CT scanning/reconstructions also facilitate clot size estimation and prompt, surgical intervention (1).

It has been suggested by some authors $(5,6)$ that since vertex bone fractures, sutural diastases or both are almost constant findings in patients with VEDHs, reconstructed thin section CT imaging (sagittal and coronal) or supplementary MR imaging is indicated when these findings are shown on routine emergency radiological examinations. Both examinations have also been suggested as the methods of choice in assessment for possible delayed haematoma formation and in cases of conservative management of VEDHs.

The treatment of VEDH ranges from conservative, twistdrill drainage to craniotomy in patients with severe clinical deterioration(5). In one case report a striking manner of treatment of the epidural clot at the vertex by percutaneous needle aspiration of communicating subgaleal haematomas is reported (10).

In our reported case there was a small right parietal haemorrhagic contusion noted. The presence of intradural haematomas or lesions worsens prognosis $(10,11)$ and probably the absence of a significant lesion contributed to the patient's good outcome. It has been reported that appearance of subarachnoid haemorrhage and other intracerebral haematomas may cause slow increase of intracranial pressure without deterioration of the clinical picture at the beginning with subsequent sudden death (12).

The decision in favour of surgical or conservative treatment is based on the calculated volume of haematoma and changes inclinical picture. As in other small epidural haematomas in clinically improving patients, managementmay consist of close observation only (2). Spontaneous recovery with non-surgical management occurred in 6 out 32 patients with VEDH reviewed by Columella et al (10).

The sagittal sinus was inferiorly displaced about $3 \mathrm{~cm}$ from the inner table of the skull in our patient. Probably there should be a critical distance between the sinus and the inner table of the calvarium to be able to have an absolute indication for evacuation. Our patient presented with neurological deficits with decerebrate upper limbs, GCS of less than 8 and with the large epidural haematoma noted on the CT scan of the brain, thus the obvious indication for ICU admission and emergency craniotomy. 
The surgical approach has sometimes not been clear and in our reported case the surgeon designed a biparietal incision both opening from the midline sagittal plane and scalp flaps, raised laterally. A biparietal free bone flap was raised taking note of the compressed dura and its sagittal sinus. Two burr holes were drilled bilaterally in the lateral aspect, sagittal suture line anteriorly and posteriorly on the free bone flap and used for hitching the dura along the suture line. This elevated the dura once complete evacuation was performed and good haemostasis achieved.

However the difference in reported cases was their approach by leaving a 'keel' of skull bone in the middle sagittal suture line that is then used for hitching the dura (1). In our reported case, the surgeon performed a different technique that worked and would be easier in a set up where there is no drill and craniotome for ease of surgery and keel bone flap formation. Other reported surgeries have also been performed with the scalpincision done coronally and skin flaps pulled anteriorly and posteriorly to expose the skull after which surgical approach would depend on the cause and extent of the bleed. The patient performed well and was discharged uneventfully on antiepileptics. He recovered fully without any neurological deficits. Subsequent follow-up was unremarkable.

In conclusion, we recognise that VEDH is a rare diagnosis that is difficult to suspect clinically. Westress the importance of imaging of head trauma patients to include the vertex on CT scans and the value of coronal and sagittal reformatted images even in the absence of neurological symptoms and especially in the presence of sutural diastases or fractures at the vertex. We also emphasize the role of MRI in this diagnostic challenge. Once the diagnosis is confirmed, there is need for rapid surgical intervention especially in patients with progressive symptoms. The choice of surgical approach should aim at achieving adequate evacuation of haematoma while at the same time aim for good hitching of dura and peridural superior sagittal sinus to prevent reaccumulation.

\section{REFERENCES}

1. Saffavi-Abbasi, S., Feiz-Erfan, I., et al. Vertex epidural hematoma manifesting with monoplegia: Case report. Barrow Quarterly. 2002; 18, 2: 25-28.

2. Guha, A., Perrin, R. G., Grossman, H., et al. Vertex epidural hematomas. Neurosurg. 1998; 25: 824- 828.

3. Alexander, G. L. Extradural haematoma at the vertex. J. Neurol. Neurosurg. Psych. 1961; 24: 381-384.

4. Harbury, O. L., Provenzale, J. M. and Barboriak, D. P. Vertex epidural hematomas: Imaging findings and diagnostic pitfalls. Eur. J. Radiol. 2000; 36: 150-157.

5. Miller, D.J., Steinmetz, M. and McCutcheon,I.E. Vertex epidural hematoma: Surgical versus conservative management. Two case reports and review of the literature. Neurosurg. 1999; 45: 621-624.

6. Messori, A., Pauri, F., Rychlicki, F., et al. Acute posttraumatic paraplegia caused by epidural hematoma at the vertex. AJNR Am. J. Neuroradiol. 2001; 22: 1748-1749.

7. Borozone, M., Gentile, S., Perria, C., et al. Vertex epidural hematomas. Surg. Neurol. 1979; 11: 277284.

8. Server, A., Tollesson, G., et al. Vertex epidural hematoma- neuroradiological findings and management. A case report. Acta. Radiol. 2002; 43: 483-485.

9. Stevenson, G.C., Brown, H. A. and Hoyt, W. F. Chronic venous epidural hematoma at the vertex.J. Neurosurg. 1964; 21: 887-891.

10. Columella, F., Gaist, G., Piazza, G. and Caraffa, T. Extradural haematoma at the vertex. J. Neurol. Neurosurg. Psychiatry. 1968; 31: 315-320.

11. Strowitziki, M., Eymann, R., Schleifer, J. and Steudel, W. I. Vertex epidural hematoma with communicating bifrontal subgaleal hematomas treated by percutaneous needle aspiration. 2001; 35: $1-4$

12. Kuday, C., Uzan, M. and Hanci, M. Statistical analysis of the factors affecting the outcome of extradural haematomas:115 cases. Acta. Neuro-chir (Wien) 1994; 131: 203-206.

13. Balik, V. and Sulla, I. Vertex extradural haematoma with associated lesions - improving clinical course with sudden death. Conservative versus surgical treatment. Bratisl. Lek. Usty. 2007; 108: 409-413. 